\title{
20 Jahre Deutsche Einheit: Schöpferischer Aufbau
}

Pünktlich zum 20. Jahrestag der Deutschen Einheit stehen deren Kosten wieder einmal im Zentrum der öffentlichen Debatte: Das Bundesverfassungsgericht hat die verfassungsrechtliche Zulässigkeit des Solidaritätszuschlags bestätigt, nachdem ein Normenkontrollantrag des Niedersächsischen Finanzgerichts inn infrage gestellt hatte. Dabei kommen aus dieser Steuerquelle nur Bruchteile der Einheitskosten. Hätte die Bankenkrise den Staatshaushalt nicht so stark belastet, könnte problemlos auf diese Einnahme verzichtet werden. Tatsächlich betragen die kumulierten Transferleistungen des Westens an den Osten derzeit rund 1,4 Billionen Euro. Das Fehlen flächendeckend „blühender Landschaften“, die doch angeblich versprochen waren - und die es auch im Westen bekanntlich nicht überall gibt -, wird gerne als Beleg für ein weitgehendes Scheitern des Aufbauprozesses genannt ebenso wie Arbeitslosigkeit und Entvölkerung. Aber waren derartige Prophezeiungen überhaupt gerechtfertigt? Was war zu erwarten, wie waren die Startbedingungen in die Marktwirtschaft?

Die 1980er Jahre waren eine Zeit inneren ökonomischen Zerfalls der DDR: den Fachleuten bekannt, von Geheimdiensten analysiert, von der Politik nicht zur Kenntnis genommen. Wichtige Auslöser waren u.a. die fundamentalen Verschiebungen in der Weltwirtschaft als Folge der Ölpreiskrise, die übermäßigen Rüstungskosten des Warschauer Pakts, schließlich aber auch die Konzentration der gewerblichen Wirtschaft auf rund 150 zentral gesteuerte Kombinate und die Folgen der Enteignungen des gewerblichen Mittelstands in den 1970er Jahren, durch die wichtige Exportmärkte vor allem im innerdeutschen Handel wegbrachen. Der „Strauß-Kredit“ des Jahres 1982 war eine Zwischenfinanzierung, die die wirtschaftlichen Folgen zeitweise verschleierte. Der Schürer-Bericht aus dem Spätsommer 1989 bot der DDR-Regierung drei „Sanierungspfade“ an: Das Land solle entweder den Wohlstand um ein Drittel absenken, die Exporte vervielfachen oder das Wirtschaftssystem aufgeben. Letzteres geschah dann auch. Mit der Währungsunion gab es für die ehemaligen Partnerländer erst recht keinen Anreiz mehr, in den neuen Bundesländern einzukaufen, obwohl dies ursprünglich für selbstverständlich gehalten wurde. Insbesondere der Sowjetunion fehlten durch den Zusammenbruch auch die Mittel, die von ihr im Rahmen der „2+4-Gespräche“ durchgesetzten langfristigen Lieferbeziehungen aufrechtzuerhalten. Dies zerstörte die letzte Hoffnung, mit einer "Ostorientierung" der gewerblichen Wirtschaft den industriellen Übergang zu meistern.

Bei der Transformation einer zentralistischen Zwangswirtschaft in eine soziale Marktwirtschaft stehen schwerwiegende Entscheidungen an über die Art der Privatisierung, die Zuordnung des neuen Privateigentums und schließlich auch die entsprechende Verfügungsgewalt. Insbesondere besteht ein Zielkonflikt zwischen Privatisierung und Sanierung. Das Zerschlagen der Kombinate folgte der an sich richtigen Grundidee, dass nur Private mit Blick auf die Marktpotenziale eine vernünftige Sanierung bewerkstelligen können, die dann aber durch Subventionen zu begleiten war. Damit wurden Bedingungen geschaffen, die auch heute noch in den neuen Bundesländern spürbar sind, nämlich einerseits strukturbestimmende verlängerte Werkbänke als Großbetriebe und andererseits eine im Vergleich zum Westen erhöhte Kleinteiligkeit ohne eigene Systemfähigkeit. Somit fehlen Führungszentralen, Forschungs- und Entwicklungskapazitäten, Wertschöpfungstiefe und Exportorientierung. Die Vorstellung, natürliches Wachstum würde diese Strukturfehler schnell heilen, bestätigte sich letztlich nicht. Damit stößt die ostdeutsche Wirtschaftsstruktur an eine - bezogen auf den Westen - relative Wachstumsgrenze: eine „70\%-Öko-

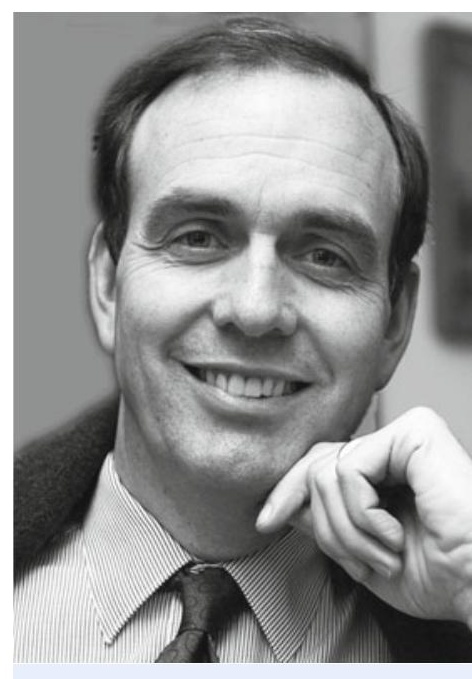

Ulrich Blum ist Präsident des Instituts für Wirtschaftsforschung Halle. 
nomie" hat sich etabliert. Die Erwerbstätigenquote hat bereits ein Niveau von $80 \%$ erreicht; dies hilft aber nicht weiter angesichts den gegenüber dem Westen längeren Arbeitszeiten und geringeren Teilzeitquoten.

Die neuen Unternehmer vor Ort hatten nur die Chance, an alte Innovationstraditionen anzuknüpfen, um die weitgehend verteilten Weltmärkte über technologische Nischen zu erobern. Denn die Alternative einer Billigstrategie zur Markteroberung war angesichts der hohen Löhne unmöglich und ein Zurückziehen auf lokale Märkte hätte unter den Beschränkungen der lokalen Kaufkraft gelitten. Viele Qualifizierte wanderten in den Westen ab, weil der Übergang und die massiven Rationalisierungsanstrengungen zunächst die Berufschancen vor Ort begrenzten, und trugen im Westen in erheblichem Maße zur gesamtdeutschen Wirtschaftsleistung bei - der wichtigsten Quelle, die Kosten der Einheit zu bewältigen und auch die Staatsschulden nicht explodieren zu lassen. Aus etwa 4,5 Mio. Menschen, die in den Westen und etwa 2,7 Mio. Menschen, die in den Osten wanderten, errechnet sich ein Saldo von rund 1,8 Mio. Personen: diese Gruppe ist überdurchschnittlich jung, qualifiziert sowie weiblich, und sie trägt gegenwärtig mit rund 60 Mrd. Euro zur deutschen Wirtschaftsleistung bei, das ist rund ein Viertel des Wachstums im Westen seit der Wende.

Bisher konnte kein Trendwachstum in den neuen Ländern erzeugt werden, das nach den Anfangserfolgen die Lücke zu Westdeutschland zu schließen erlaubt. Tatsächlich setzt sich das alte Trendwachstum der DDR aus den 1950er Jahren nach den Einbrüchen ab den 1970er Jahren fort. Das Aufholen auf das 100\%-Westniveau erscheint unter diesen Bedingungen unmöglich. Auch die weniger dichten Agglomerationsstrukturen, also das Fehlen großer Wirtschaftszentren im Osten, sprechen dagegen; sie legen nahe, dass mit der gegenwärtigen Wirtschaftsstruktur kaum mehr als $85 \%$ des westdeutschen Niveaus erreichbar ist.

Für den weiteren Aufstieg muss sich die Wirtschaft strukturell ändern. Denn die Gegenüberstellung ostdeutscher Unternehmen mit entsprechenden „Zwillingen“ im Westen zeigt, dass diese weitgehend ähnliche Leistungskennzahlen wie Produktivität und Forschungs- und Entwicklungsintensitäten besitzen. Insofern dürfen die vorhandenen Unternehmen nicht einfach durch Subventionen weiter ausgebaut werden, wie das eine „Gießkannenförderung“ tut. Das bereits sichtbare ostdeutsche Technologietrajektorium gilt es zu nutzen, das in einigen Regionen auf der Grundlage von Spitzentechnologien sichtbar wird und das hilft, die strukturellen Defizite zu überwinden. Dies bedeutet: mehr Endkunden und weniger Vorleistungsorientierung; mehr Export- und weniger Binnenabsatz; größere, durch internes Wachstum oder Zusammenschlüsse erhöhte Schlagkraft; Konsolidieren von Märkten - auch eine Notwendigkeit bei der Übergabe der Unternehmen in die nächste Generation, die verstärkt ansteht. Letztlich lehrt alle wirtschaftshistorische Erfahrung, dass derart massive Transformationen wie in Mittel- und Osteuropa mehr als eine Generationen benötigen, um erfolgreich zum Abschluss gebracht zu werden.

Die bisher erreichte Leistungsfähigkeit geht vor allem auf das qualifizierte Humankapital zurück, und dieses muss weiter genutzt und entwickelt werden. Der zunehmende Fachkräftebedarf wird zu einer außerordentlichen Herausforderung, der sich die Unternehmen stellen müssen: durch steigende und globale Attraktivität des Unternehmens als Arbeitgeber. Industrielle und auch kulturelle Traditionen der Region tragen damit zur internen und externen Identifikationsstiftung bei. Deshalb macht sich insbesondere im mitteldeutschen Wirtschaftsraum durchaus Optimismus breit, dass die kommenden zehn Jahre gute Jahre werden können, weil die 